\title{
Novel therapeutic strategies for patients with triple-negative breast cancer
}

REVIEW

This article was published in the following Dove Press journal:

OncoTargets and Therapy

21 October 2016

Number of times this article has been viewed

Jun-Fei Zhang'

Jia Liu',

Yu Wang ${ }^{1,2}$

Bin Zhang ${ }^{1,2}$

'Affiliated Hospital of Inner Mongolia University for the Nationalities,

Tongliao, Inner Mongolia, People's

Republic of China; ${ }^{2}$ Medicinal

Chemistry and Pharmacology

Institute, Inner Mongolia University

for the Nationalities, Tongliao, Inner

Mongolia, People's Republic of China
Correspondence: Bin Zhang

Medicinal Chemistry and Pharmacology

Institute, Inner Mongolia University

for the Nationalities, No 1742 Holin

He Street, Tongliao, Inner Mongolia,

Autonomous Region, 028007, People's

Republic of China

Tel +86475 83। 4245

Emailbzh99II@I63.com
Abstract: Triple-negative breast cancer (TNBC) represents a very heterogeneous group of breast diseases. Currently, the backbone of therapy for TNBC is mainly chemotherapy as there are no effective specific targeted agents approved to treat TNBC. Despite initial responses to chemotherapy, resistance frequently and rapidly develops and metastatic TNBC has a poor prognosis. Therefore, new targeted strategies are, accordingly, urgently needed. This article discusses the recent developments in targeted agents explored for TNBC, aiming to offer novel therapeutic strategies that can potentially assist in designing personalized therapeutics in the future as well as provide the basis for further research in an attempt to target TNBC.

Keywords: therapeutic strategies, TNBC, targeted agents

\section{Introduction}

Breast cancer $(\mathrm{BC})$ is one of the most common cancers for females and the leading cause of cancer-related death in females globally, with a high incidence rate and mortality. ${ }^{1}$ "Triple-negative" BC (TNBC) was mentioned for the first time in 2005, and, since then, this term has been appearing in publications. ${ }^{2} \mathrm{TNBC}$, identified as a clinically important subgroup of BC in the early 2000s and characterized by an especially poor prognosis, ${ }^{3}$ is an aggressive $\mathrm{BC}$ subtype lacking the expression of estrogen receptor (ER) and progesterone receptor and overexpression or gene amplification of human epidermal growth factor receptor 2 (HER2). TNBC accounts for a disproportionate number of deaths from $\mathrm{BC}$, especially among premenopausal African-American and Hispanic women who have younger age, more advanced stage distribution, and higher incidence rates. ${ }^{4-6}$

Disease dynamics, risk of recurrence, and patterns of metastasis in TNBC are different from other subtypes of BC. The most favorable prognosis of TNBCs is predominantly determined by a good response to (neo)adjuvant chemotherapy (NAC), as defined by a pathological complete response ( $\mathrm{pCR}$ ), as a surrogate end point for long-term outcomes. ${ }^{7,8}$ Indeed, patients who respond poorly to NAC are significantly correlated with unfavorable prognosis. ${ }^{7}$ Patients who do not achieve pCR still have a high risk of early relapse in the 2 years following surgery; for instance, distant relapse more often involves extra-skeletal sites than bones, in spite of the use of chemotherapy. ${ }^{9}$ Unlike other subtypes, there are currently no effective specific targeted agents approved to treat TNBC, and the only option for patients is systemic chemotherapy with its inherent toxicities. ${ }^{10}$

$\mathrm{BC}$ represents a very heterogeneous group of breast diseases, and this heterogeneity has extended beyond the classic divisions of ER, progesterone receptor, and HER2. TNBC constitutes $\sim 10 \%-20 \%$ of all BC cases. ${ }^{6}$ The term TNBC could also be often called "basal-like (BL) BC"; however, they are not the same. BL BC 
belongs to a subtype of TNBC and accounts for $47 \%-88 \%$ of all TNBCs. ${ }^{11,12}$ Modern treatment strategies are tailored to molecular subtypes. ${ }^{13}$ To better understand the complexity of TNBC and to develop effective therapeutic strategies against it, more extensive genomic, molecular, and biological analyses of TNBCs in many studies classify it into diverse subtypes. According to TNBCs displaying different gene expression and gene ontologies, Lehmann et al $^{11}$ identified six TNBC subtypes, consisting of two BL (BL1 and BL2), an immunomodulatory, a mesenchymal (MES), an MES stemlike, and a luminal androgen receptor (LAR) subtype, and the distinct subtypes had differential sensitivity to targeted therapies. Subsequently, TNBC using mRNA expression and DNA profiling is defined as four stable TNBC subtypes: LAR, MES, BL immune suppressed, and BL immune activated. ${ }^{12}$ Regardless of identifying TNBC subtypes, subtype-specific molecularly targeted strategies have provided the foundation for more effective treatment.

As of today, TNBC is associated with an observably higher odds of relapse and poorer overall survival (OS) in the first few years after diagnosis, compared with other BC subtypes, despite its usual high chemotherapy sensitivity. ${ }^{14}$ The absence of clinically efficient molecularly targeted therapy is still of great concern in the treatment of patients with TNBC. Predisposition gene mutations, including poly (ADP-ribose) polymerase (PARP), ${ }^{15}$ vascular endothelial growth factor (VEGF), ${ }^{16}$ and epidermal growth factor receptor (EGFR), ${ }^{17}$ especially germline genetic testing of BC type 1 susceptibility protein (BRCA1) and BC type 2 susceptibility protein (BRCA2) mutation, are observed among a large TNBC cohort. BRCA1 and BRCA2 have a clear role for patients with TNBC, and BC in carriers with germline BRCA1 mutations is more likely to be TNBC as opposed to $15 \%-20 \%$ (reflective of the general population) of BRCA2 carriers. ${ }^{18-20}$ The identification of molecular targeted agents will be critical to improving survival in the triple-negative (TN) study population. This review discusses the recent discoveries that have furthered our understanding of TNBC and offers a basis for further research for future treatments.

\section{The target and the treatment of TNBC}

\section{Traditional chemotherapy for TNBC}

Currently, the response to chemotherapy in patients with TNBC may be good, although there is a lack of options. In a meta-analysis of five adjuvant trials comparing anthracycline-containing regimens with cyclophosphamide, methotrexate, and fluorouracil (CMF), a superiority of anthracycline-containing regimens over CMF has been demonstrated via HER2-negative deriving benefit from anthracyclines. ${ }^{21}$ However, the addition of epirubicin to CMF, in a randomized Phase III study, tends to be more superior than $\mathrm{CMF}$ alone in the 5-year disease-free survival and 5-year OS for patients with TNBC. ${ }^{22}$ With regard to the use of taxane-based adjuvant regimens, a meta-analysis of randomized trials has evaluated questions about the efficacy of incorporating taxanes into anthracycline-based regimens in terms of disease-free survival and OS, independently of ER expression for early BC. ${ }^{23}$ Therefore, currently, anthracycline/taxane-based adjuvant regimens tend to be clinically the most appropriate adjuvant therapy for patients with TNBC. Exploratory analysis has showed the efficacy of anthracycline/taxane-based adjuvant regimens in combination with capecitabine treatment in a subset of 202 patients with TNBC enrolled in the FinXX study. ${ }^{24}$ The addition of epothilone B analog ixabepilone to capecitabine, which results in an approximately twofold increase in median progression-free survival (PFS) for TN patients, is being compared with capecitabine alone. ${ }^{25}$ Chemotherapy remains the backbone of therapy for TN disease, especially in the metastatic setting, but continued therapy with chemotherapy is shown to encourage tumor resistance to these agents.

\section{Targeted therapy and novel systemic therapy for TNBC}

Despite initial responses to anthracycline/taxane-based adjuvant regimens, TNBCs often recur with chemotherapyresistant, visceral, and brain metastasis. Therefore, targeted agents that are fundamentally different from these classes of regimens need to be explored in TNBC research. In addition, on the basis of these, patients with TNBC need to be best treated with novel therapeutic alternatives, such as third-generation adjuvant chemotherapy and NAC, for instance. ${ }^{26}$

\section{PARP inhibitors}

DNA frequently harbors diverse lesions, including singlestrand breaks, double-strand breaks, and homologous recombination (HR). ${ }^{27}$ The PARPs are a large family of enzymes with diverse functions, some of which are important for the repair of single-strand breaks in DNA via the base excision repair pathway. ${ }^{28}$ BRCA1 or BRCA2 dysfunction resulted in a lack of HR and markedly sensitized cells to inhibition of PARP enzymatic activity. ${ }^{29}$ Inhibiting PARP in HR-deficient cells leads to the accumulation of double-strand breaks, genomic instability, and specific synthetic lethality. ${ }^{30}$ 
When some factors, such as chemotherapeutic agents, cause DNA damage, PARP could repair DNA damage and PARP inhibitors might sensitize TNBC to these agents. ${ }^{31}$ The role of PARPs in DNA repair prompted the investigation of PARP inhibitors as potential treatments for cancer, a disease in which DNA replication and, therefore, replication errors are prominent features, and deficiencies in DNA repair pathways are common.

PARP inhibitors, targeting DNA repair deficiencies, so far represent a real milestone in the treatment of advanced TNBC with BRCA gene dysfunction. ${ }^{32,33}$ Currently, several PARP inhibitors such as olaparib, veliparib, rucaparib, niraparib, and talazoparib (BMN673) are undergoing clinical development. Olaparib (AZD2281) is a well-tolerated oral PARP inhibitor, which has shown promising monotherapy activity in patients with BRCA1 or BRCA2 mutations who have breast and ovarian cancers. ${ }^{34,35}$ Gelmon et $\mathrm{al}^{36}$ have shown, for the first time, that olaparib has activity responses in patients with high-grade serous cancer without germline BRCA1 or BRCA2 mutations, which has illustrated that olaparib is a promising therapeutic option for women with these aggressive cancers. In addition, iniparib (BSI-201) failed to possess characteristics typical of PARP inhibitors, but could suppress genes involved in telomere function and nonselectively modified cysteine-containing proteins in tumor cells, which may be a result of blockade of other PARP family members. ${ }^{37-39}$ Compared with iniparib, olaparib was more potent in the inhibition of $\mathrm{BC}$ cell growth. ${ }^{40}$

Considering preclinical observations regarding the actions of PARP inhibitors, patients with BRCA mutations are the most investigated population in clinical trials of different PARP inhibitors. The combination treatment of PARP inhibitors and chemotherapy or targeted therapy has more potential advantages for the treatment of TNBC. To date, a few Phase I-III trials (Table 1) have demonstrated the efficacy of PARP inhibitors in patients who have TNBC, in parallel in the metastatic and neoadjuvant settings. As part of the I-Spy 2 trial, the combination of carboplatin and veliparib added to weekly paclitaxel and followed by doxorubicin/cyclophosphamide led to a doubling of the $\mathrm{pCR}$ rate in patients with TNBC (from $26 \%$ to $52 \%$ ). A Phase III trial statistics for this combination predicted a probability of success of $90 \% .{ }^{41}$ Combined treatment with olaparib and either the CDK1 inhibitor (RO-3306) or a pan HER inhibitor (neratinib, afatinib) resulted in superior growth inhibition compared to that obtained with olaparib alone. ${ }^{40}$ Although better patient stratification is likely to improve therapeutic outcome, several PARP inhibitor resistance mechanisms in BRCA-associated tumors have been proposed..$^{42}$ Long-term treatment with olaparib did result in the development of drug resistance, caused by upregulation of $\mathrm{Abcb} 1 \mathrm{a} / \mathrm{b}$ genes encoding P-glycoprotein efflux pumps. Indeed, the mechanism of acquired resistance to olaparib could be effectively circumvented by an ideal P-glycoprotein drug efflux pump inhibitor, tariquidar (XR9576). ${ }^{33,43}$ More insight into the mechanisms of PARP inhibitor resistance will therefore be instrumental to develop the most optimal treatment strategies that prevent or counteract resistance to PARP inhibitors. ${ }^{42}$

\section{Platinum salts}

Platinum salts (cisplatin and carboplatin) are also important DNA-damaging agents with activity in BC, particularly in the TN subgroup. ${ }^{50}$ The strong interest in platinum-based therapies derives from the fact that almost all TNBCs belong to the molecular subgroup of BLs according to the Perou classification. ${ }^{51}$ Table 2 summarizes the current clinical trials investigating platinum salts in BC. A total of 28 studies (six randomized controlled trials and 22 retrospective or prospective studies) have shown that the pooled pCR rate was $45 \%$ when a total of 1,598 patients with TNBC were treated with platinum-based NAC. In randomized trials, pCR rate with NAC containing platinum drugs was significantly increased

Table I Active large Phase I-III trials of PARP inhibitors in patients with TNBC

\begin{tabular}{lllll}
\hline $\begin{array}{l}\text { ClinicalTrials.gov } \\
\text { identifier }\end{array}$ & Population & Therapy & Phase & Status \\
\hline NCT02338622 & Advanced TNBC & Olaparib (AZD228I) + AZD5363 (AKT inhibitor) & I & Recruiting ${ }^{44}$ \\
NCT00707707 & mTNBC & AZD228I + paclitaxel & I & Active, not recruiting ${ }^{45}$ \\
NCT01074970 & TNBC with BRCAI/2 mutations & Cisplatin + rucaparib + preoperative chemotherapy & II & Active, not recruiting ${ }^{46}$ \\
NCT02032277 & Early TNBC & Carboplatin-based NAC + veliparib/placebo & III & Recruiting $^{47}$ \\
NCT0I204I25 & TNBC & Iniparib (SAR2405550-BSI-20I) + paclitaxel & II & Recruiting $^{48}$ \\
NCT00938652 & mTNBC & BSI-20I + gemcitabine/carboplatin & III & Completed $^{49}$ \\
\hline
\end{tabular}

Note: Recent preclinical and clinical data indicate that iniparib does not possess characteristics typical of PARP inhibitor class.

Abbreviations: BRCA, breast cancer susceptibility protein; NAC, neoadjuvant chemotherapy; PARP, poly(ADP-ribose) polymerase; TNBC, triple-negative breast cancer; mTNBC, metastatic TNBC. 
Table 2 Reported studies evaluating cisplatin or carboplatin for the treatment of patients with TNBC

\begin{tabular}{|c|c|c|c|c|}
\hline $\begin{array}{l}\text { ClinicalTrials.gov } \\
\text { identifier }\end{array}$ & Population & Therapy & Phase & Status \\
\hline NCT01930292 & BL/claudin-low TNBC & Paclitaxel + carboplatin & I & Active, not recruiting ${ }^{57}$ \\
\hline NCT0I982448 & TNBC & Cisplatin + paclitaxel & II & Recruiting ${ }^{58}$ \\
\hline NCT0I560663 & TNBC & Docetaxel + carboplatin & I & Recruiting 59 \\
\hline NCT02393794 & $\mathrm{mTNBC}$ & Romidepsin + cisplatin & $\mathrm{I} / \mathrm{II}$ & Recruiting 60 \\
\hline NCT01216124 & Local advanced TNBC & Docetaxel + oxaliplatin & II & Unknown ${ }^{61}$ \\
\hline NCTOI 276769 & TNBC & Paclitaxel + carboplatin/epirubicin & II & Unknown ${ }^{62}$ \\
\hline NCT0I2I6III & TNBC & Paclitaxel + cisplatin & III & Unknown ${ }^{63}$ \\
\hline NCT0244539I & BL TNBC & Platinum-based chemotherapy & III & Recruiting $^{64}$ \\
\hline NCT0086I 705 & TNBC & $\begin{array}{l}\text { Carboplatin + bevacizumab + paclitaxel + } \\
\text { doxorubicin + cyclophosphamide }\end{array}$ & II & Active, not recruiting, has results ${ }^{65}$ \\
\hline
\end{tabular}

Abbreviations: BL, basal like; TNBC, triple-negative breast cancer; mTNBC, metastatic TNBC.

in contrast to nonplatinum agents (risk ratio $=1.45 ; 95 \% \mathrm{CI}$, 1.25-1.68; $P<0.0001)$. Compared with non-TN, TNBCs treated with NAC containing cisplatin or carboplatin were associated with a threefold increase in the rate of pCR. ${ }^{52}$ Olaparib in combination with platinum drugs increased the recurrence-free survival and OS. Therefore, platinum analogs alone or combined with targeted agents such as PARP inhibitors might clinically guide the design of future BRCA1-associated BC trials. Gemcitabine and carboplatin plus iniparib as neoadjuvant therapy improved the clinical benefit rate, median PFS, and median OS versus gemcitabine and carboplatin (GC) alone. ${ }^{53}$ Gemcitabine and carboplatin plus iniparib in Phase II and III clinical trials were also preoperatively active in early TNBC and led to an increased clinical benefit rate, in contrast to the combination of GC..$^{32,54,55}$ The addition of carboplatin to taxane-anthracycline chemotherapy plus targeted therapy substantially increased pCRs in patients with stage II-III TNBC. ${ }^{56}$

\section{Epidermal growth factor receptor}

Most TNBCs were also characterized by overexpression of EGFR, which is correlated with a poor prognosis ${ }^{66}$ and prompted a series of clinical trials incorporating anti-EGFR-directed therapies (Table 3), but the EGFR inhibitor seldom inhibited this pathway. The TBCRC001 trial has investigated not only cetuximab, a monoclonal anti-EGFR antibody as a singleagent blocked expression of the EGFR pathway alone was uncommon but also cetuximab added to a platinum agent had little activity. However, despite these discouraging results, there were long-lasting responses of $>12$ months (two even over 2.5 years) in four patients (two in the monotherapy and two in the combination arm). Experimental results from the serial biopsies of 18 patients have shown EGFR pathway activation (81\%); however, this pathway could only be inactivated by cetuximab in five patients (38\%). Clinical benefit was restricted to these patients. ${ }^{67}$ Gefitinib, the most effective EGFR inhibitor, efficiently blocked epidermal growth factor (EGF)-stimulated phosphorylation of EGFR, reduced phosphorylation of both MAPK and protein kinase B (AKT), and induced G1 arrest in the TNBC cells. Fortunately, the triple combination of gefitinib, carboplatin, and docetaxel synergistically enhanced the treatment effect in the TNBC cells. ${ }^{68}$ Elbaz et $\mathrm{al}^{69}$ have shown, for the first time, that cannabidiol significantly inhibited BC growth and metastasis through novel mechanisms by the inhibition of EGF/EGFR signaling and modulation of the tumor microenvironment, which also

Table 3 Results from selected trials with EGFR-targeted therapies in patients with TNBC

\begin{tabular}{|c|c|c|c|c|}
\hline $\begin{array}{l}\text { ClinicalTrials.gov } \\
\text { identifier }\end{array}$ & Population & Therapy & Phase & Status \\
\hline NCT00463788 & TNBC & Cetuximab + cisplatin & II & Completed, has results ${ }^{70}$ \\
\hline NCT00232505 & TNBC & Cetuximab + carboplatin & II & Active, not recruiting ${ }^{71}$ \\
\hline NCT0I097642 & TNBC & Cetuximab + ixabepilone & II & Active, not recruiting ${ }^{72}$ \\
\hline NCT02I 58507 & $\mathrm{mTNBC}$ & Veliparib + lapatinib & - & Recruiting ${ }^{73}$ \\
\hline NCTOI 732276 & mTNBC & Gefitinib & II & Not yet recruiting ${ }^{74}$ \\
\hline NCT00894504 & $\mathrm{mTNBC}$ & Gemcitabine + carboplatin + panitumumab & II & Completed, has results ${ }^{75}$ \\
\hline NCT0I426880 & TNBC & Carboplatin + NAC & II/III & Completed ${ }^{76}$ \\
\hline NCT00540358 & $\mathrm{mTNBC}$ & Gemcitabine/carboplatin + iniparib & II & Completed ${ }^{77}$ \\
\hline
\end{tabular}

Abbreviations: EGFR, epidermal growth factor receptor; NAC, neoadjuvant chemotherapy; TNBC, triple-negative breast cancer; mTNBC, metastatic TNBC. 
indicated that cannabidiol is a novel therapeutic option in highly aggressive BC subtypes including TNBC.

\section{Antiangiogenesis}

TNBC is associated with a significantly higher expression and more frequent amplification of VEGF-A. ${ }^{78,79}$ Bevacizumab, an antiangiogenic monoclonal antibody, has been shown to increase the risk ratio and PFS of patients with human EGFR2-negative metastatic BC when added to first-line chemotherapy in randomized Phase III trial. ${ }^{80,81}$ The addition of either carboplatin or bevacizumab to NAC improved pCR rates, while higher $\mathrm{pCR}$ rates did not justify the routine addition of bevacizumab in stage II to III TNBC.$^{82}$ The addition of bevacizumab to anthracycline-taxane-based chemotherapy in TNBC increased the $\mathrm{pCR}$ rate significantly, and $\mathrm{pCR}$ rates were $27.9 \%$ without and $39.3 \%$ with bevacizumab. ${ }^{83}$ In addition, the studies have also shown a significant improvement in pCR, PFS, and OS when bevacizumab was added to NAC versus chemotherapy alone. ${ }^{82,84}$ In brief, combined chemotherapy with anti-VEGF therapy may become the potential to be a significant first-line treatment option.

\section{Immune checkpoint inhibitors}

Currently, recent studies of tumor lymphocytic immune infiltrates in $\mathrm{BC}$ have suggested an improved prognosis associated with increasing levels of tumor-infiltrating lymphocytes (TIL). ${ }^{85} \mathrm{BC}$ is capable of stimulating the immune system. Furthermore, the intensity of tumor immune response has an effect on the effectiveness of cancer therapy and is correlated with favorable clinical outcome in this disease. ${ }^{86}$ It is reported that programmed death 1 ligand 1 (PD-L1), an immune checkpoint, was positively prevalent in TN status and high levels of TILs. ${ }^{87}$ Programmed cell death protein 1 (PD-1) is also an immune checkpoint that limits T-cell effector functions within tissues. When PD-L1 binds to PD-1, which is present on the surface of T-cells, the lymphocytes become inactivated. ${ }^{88}$ Atezolizumab (MPDL3280A), a monoclonal anti-PD-L1 antibody, was generally well tolerated and demonstrated promising efficacy in pretreated metastatic PD-L1 TNBC. ${ }^{87,89}$ In addition, the preliminary results of a recent study have suggested that in a Phase I clinical trial, single-agent pembrolizumab (MK-3475), a monoclonal anti-PD-1, is a well-tolerated and effective treatment with significant therapeutic activity in a subset of heavily pretreated patients with recurrent/metastatic TNBC (mTNBC).${ }^{90}$ Preliminary results of a combination of atezolizumab plus nab-paclitaxel chemotherapy in mTNBC have been reported, ${ }^{91}$ and a Phase III trial in untreated
mTNBC cancer as first-line therapy has been opened. Multiple additional immune checkpoint receptors and their ligands such as the cytotoxic T lymphocyte antigen-4 (CTLA-4) are the main targets for blockade. The monoclonal antibody, tremelimumab, which inhibits the CTLA-4 pathway, was evaluated in hormone-positive $\mathrm{BC}$ and has shown activity. ${ }^{92}$ In GeparSixto and CALGB 40603 trials, it might become clear how best to incorporate platinum into the treatment of early TNBC through biomarkers of TIL that could be evaluated as therapeutic targets. ${ }^{93,94}$ Patients with high TILs, which were highly significant pathologic predictors for anthracycline/taxane-based NAC in TNBC, have obviously improved pCR rates, particularly patients treated with carboplatin. Therefore, immunomodulatory agents might appear to increase response rates to NAC..$^{93-96}$ The immunomodulatory drugs lay the foundation for the development of immune-based therapies in TNBC in assessing the immunogenicity of TNBC, indicating a subset of patients who may benefit from immune therapy.

\section{$\mathrm{PI3K} / \mathrm{Akt} / \mathrm{mTOR}$}

An initial analysis of whole genome and transcriptome sequencing of TNBCs has suggested that many of the tumors had co-activation of MAPK and PI3K/AKT pathways. Earlier studies showed combinations via inhibiting the coactivation of these pathways had some excellent responses in some but not all patients who were more concerned in phase I trial. While MES tumors tend to frequently activate KRAS, BRAF, and RAS pathways, some may activate JAK-2/ STAT3. ${ }^{97}$ The current study has demonstrated that celastrolinduced apoptosis in TNBC cells might be mediated through mitochondrial dysfunction and PI3K/Akt axis, while PI3K/ Akt/mTOR inhibitor PF-04691502 and mTOR inhibitor, rapamycin, enhanced the effect of celastrol-induced apoptosis in TNBC cells. ${ }^{98}$ However, focusing on therapeutic targets geared toward the most commonly known activated pathways such as P53, MAPK, PI3K/AKT, Jak-2, KRAS, BRAF, and RAS and incorporating gene expression in advanced TNBC treatment.

\section{Androgen receptor}

It has been shown that androgen receptor (AR)-positive TNBCs represented a distinct BC subgroup with adverse clinical outcome, and AR blockade was regarded as a potential endocrine therapy for these patients with TNBC. ${ }^{99}$ The LAR subtypes characterized by AR expression were sensitive to the AR antagonist bicalutamide alone or in combination with PI3K inhibitors. ${ }^{11,100,101}$ 


\section{Other targets}

Low thyroid hormone receptor beta $(\mathrm{TR} \beta)$ expression was associated with enhanced resistance to specific therapeutic regimens such as docetaxel and doxorubicin in TNBC through the increase of cAMP/PKA gene signaling, but TR $\beta$-specific agonists in combination with docetaxel or doxorubicin treatment enhanced the sensitivity of TNBC cells. ${ }^{102}$ Down-regulation of IGF1R depending on focal adhesion kinase (FAK) regulated the epithelial-to-mesenchymal transition and suppressed colony formation, migration, and invasion of TNBC via the IGF1R/FAK signaling axis, suggesting that co-targeting of IGF1R and FAK could act as therapy markers for MES TNBCs through the IGF1R/ FAK signaling pathway. ${ }^{103}$ The article by Cerqueira et al ${ }^{104}$ has addressed that high levels of CIP4 expression promoted metastasis of TNBC, while knockdown of CIP4 led to had no overt effect on tumor growth but observably suppressed the incidence of TNBC cell invasion in vitro and tumor metastasis in vivo through EGFR/CIP4/Erk/MMP-2 signaling pathway, which demonstrated that targeting CIP4 will become a poor prognostic marker in TNBC. Tate et $\mathrm{al}^{105}$ have investigated that the histone deacetylase inhibitor, panobinostat (LBH589), was overtly toxic to TNBC cells in vitro due to G2/M cell cycle arrest and apoptosis, and decreased tumorigenesis in vivo and this was also an apparent partial reversal of epithelial-to-mesenchymal transition evidenced by increased cadherin-1, E-cadherin (CDH1) protein expression, and morphology changes in MDA-MB-231 cells, suggesting panobinostat acted as a promising therapeutic option for aggressive TNBC types. A Phase Ib study first reported that pembrolizumab (MK-3475), a highly selective, humanized IgG4/kappa isotype antibody, could block the PD-1 receptor ligand pathway, thereby reactivating the immune system to eradicate tumors to pretreat patients with recurrent/ mTNBC. ${ }^{106}$ A retrospective analysis demonstrated that the BRCA1 locus product, BRCA1-IRIS, silencing or inactivation using a novel inhibitory mimetic peptide could sensitize TNBC cells to low paclitaxel concentrations in vitro and in vivo, through inactivating two autocrine signaling loops involving EGFR-1 and EGFR3 that regulated AKT causing survivin degradation and Fork head box O3a (FOXO3a) upregulation. However, BRCA1-IRIS targeting therapy cannot completely eradicate TNBC cells overexpressing BRCA1-IRIS; several inhibitors must be combined, such as ERK1/2, AKT, and/or BRCA1-IRIS inhibitors. ${ }^{107}$ These combined therapies allow for a more individualized strategic therapy in the future, as well as provide the basis for further research in an attempt to target TNBC.

\section{Prospect}

Due to the rapid social development and the increasing of people's life pressure, patients in growing numbers were diagnosed with breast cancer. ${ }^{1,108}$ Along with the increase in patients with $\mathrm{BC}$, early treatment and early diagnosis were advocated. Once diagnosed, identification of targeted therapy is of great significance through differences in the genetic profile of primary BC. ${ }^{10,108}$ Moreover, participation in an exercise and diet counseling program would lead to loss of body fat, improved fitness, and quality of life and increase habitual physical activity in survivors of TNBC. ${ }^{109,110}$ In addition, patients with TNBC undergoing breast-conserving therapy had better outcomes than with modified radical mastectomy. ${ }^{111}$ Reasonable use of these drugs about the above targeted drugs is increasingly utilized for all BC subtypes, because of their advantages, including higher rates of breastconserving surgery and the possibility of measuring early in vivo response to systemic treatment. ${ }^{12}$

\section{Conclusion}

The targeted therapy drugs could become the main content of research currently and in the future. PARP inhibitors and platinum salts might, in an even better fashion, be incorporated into other drugs for clinical treatment of TNBC.

\section{Acknowledgment}

This work was support by the National Natural Science Foundation of China (No 81373573).

\section{Disclosure}

The authors report no conflicts of interest in this work.

\section{References}

1. Siegel R, Ma J, Zou Z, Jemal A. Cancer statistics, 2014. CA Cancer J Clin. 2014;64(1):9-29.

2. Brenton JD, Carey LA, Ahmed AA, Caldas C. Molecular classification and molecular forecasting of breast cancer: ready for clinical application? J Clin Oncol. 2005;23(29):7350-7360.

3. Malorni L, Shetty PB, De Angelis C, et al. Clinical and biologic features of triple-negative breast cancers in a large cohort of patients with long-term follow-up. Breast Cancer Res Treat. 2012;136(3): 795-804.

4. Lara-Medina F, Pérez-Sánchez V, Saavedra-Pérez D, et al. Triple-negative breast cancer in Hispanic patients. Cancer. 2011;117:3658-3669.

5. Amirikia KC, Mills P, Bush J, Newman LA. Higher population-based incidence rates of triple-negative breast cancer among young AfricanAmerican women. Cancer. 2011;117:2747-2753.

6. Morris GJ, Naidu S, Topham AK, et al. Differences in breast carcinoma characteristics in newly diagnosed African-American and Caucasian patients. Cancer. 2007;110:876-884

7. von Minckwitz G, Untch M, Blohmer JU, et al. Definition and impact of pathologic complete response on prognosis after neoadjuvant chemotherapy in various intrinsic breast cancer subtypes. J Clin Oncol. 2012;30(15):1796-1804. 
8. Cortazar P, Zhang L, Untch M, et al. Pathological complete response and long-term clinical benefit in breast cancer: the CTNeoBC pooled analysis. Lancet. 2014;384(9938):164-172.

9. Groheux D, Giacchetti S, Delord M, et al. Prognostic impact of 18FFDG PET/CT staging and of pathological response to neoadjuvant chemotherapy in triple-negative breast cancer. Eur J Nucl Med Mol Imaging. 2015;42(3):377-385.

10. Ismail-Khan R, Minton S, Khakpour N. Triple-Negative Breast Cancer/ Breast Disease. New York: Springer; 2015:463-472.

11. Lehmann BD, Bauer JA, Chen X, et al. Identification of human triplenegative breast cancer subtypes and preclinical models for selection of targeted therapies. J Clin Invest. 2011;121(7):2750-2767.

12. Krings G, Nystrom M, Mehdi I, Vohra P, Chen YY. Diagnostic utility and sensitivities of GATA3 antibodies in triple-negative breast cancer Hum Pathol. 2014;45(11):2225-2232.

13. Kravchenko J, Akushevich I, Seewaldt VL, Abernethy AP, Lyerly HK Breast cancer as heterogeneous disease: contributing factors and carcinogenesis mechanisms. Breast Cancer Res Treat. 2011;128(2): 483-493.

14. Kassam F, Enright K, Dent R, et al. Survival outcomes for patients with metastatic triple-negative breast cancer: implications for clinical practice and trial design. Clin Breast Cancer. 2009;9(1):29-33.

15. Dantzer F, de La Rubia G, Ménissier-De Murcia J, Hostomsky Z, de Murcia G, Schreiber V. Base excision repair is impaired in mammalian cells lacking Poly(ADP-ribose) polymerase-1. Biochemistry. 2000;39(25): 7559-7569.

16. Miller JW, Le Couter J, Strauss EC, Ferrara N. Vascular endothelial growth factor a in intraocular vascular disease. Ophthalmology. 2013 120(1):106-114.

17. Sutton LM, Han JS, Molberg KH, et al. Intratumoral expression level of epidermal growth factor receptor and cytokeratin 5/6 is significantly associated with nodal and distant metastases in patients with basallike triple-negative breast carcinoma. Am J Clin Pathol. 2010;134(5) 782-787

18. Kandel MJ, Stadler Z, Masciari S, et al. Prevalence of BRCA1 mutations in triple negative breast cancer (BC). J Clin Oncol. 2006;24:508.

19. Somer R, Farengo-Clark D, Patel A, Schwarting R, Grana G, Rodler E. Screening for BRCA1 mutations in patients with triple negative breast cancer and basal phenotype. Cancer Res. 2009;69:4075-4075.

20. Couch FJ, Hart SN, Sharma P, et al. Inherited mutations in 17 breast cancer susceptibility genes among a large triple-negative breast cancer cohort unselected for family history of breast cancer. J Clin Oncol. 2015;33(4):304-311

21. Di Leo A, Desmedt C, Bartlett JM, et al. Final results of a meta-analysis testing HER2 and topoisomerase II \{alpha\} genes as predictors of incremental benefit from anthracyclines in breast cancer. J Clin Oncol. 2010;28:519

22. Rocca A, Paradiso A, Sismondi P, et al. Benefit from CMF with or without anthracycline(A) in relation to biologic profiles in early breast cancer. J Clin Oncol. 2011;29:1031.

23. De Laurentiis M, Cancello G, D'Agostino D, et al. Taxane-based combinations as adjuvant chemotherapy of early breast cancer: a metaanalysis of randomized trials. J Clin Oncol. 2008;26(1):44-53.

24. Jiang Y, Yin W, Zhou L, et al. First efficacy results of capecitabine with anthracycline- and taxane-based adjuvant therapy in high-risk early breast cancer: a meta-analysis. PLoS One. 2012; 7(3):e32474

25. Perez EA, Patel T, Moreno-Aspitia A. Efficacy of ixabepilone in ER/ PR/HER2-negative (triple-negative) breast cancer. Breast Cancer Res Treat. 2010;121(2):261-271.

26. Liedtke C, Mazouni C, Hess KR, et al. Response to neoadjuvant therapy and long-term survival in patients with triple-negative breast cancer. $J$ Clin Oncol. 2008;26(8):1275-1281.

27. Hoeijmakers JH. Genome maintenance mechanisms for preventing cancer. Nature. 2001;411(6835):366-374.

28. Amé JC, Spenlehauer C, de Murcia G. The PARP superfamily. Bioessays. 2004;26(8):882-893.
29. Bryant HE, Schultz N, Thomas HD, et al. Specific killing of BRCA2deficient tumours with inhibitors of poly (ADP-ribose) polymerase. Nature. 2005;434(7035):913-917.

30. Farmer H, McCabe N, Lord CJ, et al. Targeting the DNA repair defect in BRCA mutant cells as a therapeutic strategy. Nature. 2005; 434(7035):917-921.

31. Ljungman M. Targeting the DNA damage response in cancer. Chem Rev. 2009;109:2929-2950.

32. Metzger-Filho O, Tutt A, de Azambuja E, et al. Dissecting the heterogeneity of triple-negative breast cancer. J Clin Oncol. 2012;30(15):1879-1887.

33. Rottenberg S, Jaspers JE, Kersbergen A, et al. High sensitivity of BRCA1-deficient mammary tumors to the PARP inhibitor AZD2281 alone and in combination with platinum drugs. Proc Natl Acad Sci US A. 2008;105(44):17079-17084.

34. Audeh MW, Carmichael J, Penson RT, et al. Oral poly(ADP-ribose) polymerase inhibitor olaparib in patients with BRCA1 or BRCA2 mutations and recurrent ovarian cancer: a proof-of-concept trial. Lancet. 2010;376(9737):245-251.

35. Tutt A, Robson M, Garber JE, et al. Oral poly(ADP-ribose) polymerase inhibitor olaparib in patients with BRCA1 or BRCA2 mutations and advanced breast cancer: a proof-of-concept trial. Lancet. 2010 376(9737):235-244

36. Gelmon KA, Tischkowitz M, Mackay H, et al. Olaparib in patients with recurrent high-grade serous or poorly differentiated ovarian carcinoma or triple-negative breast cancer: a phase 2, multicentre, open-label, non-randomised study. Lancet Oncol. 2011;12(9):852-861.

37. Ji J, Lee MP, Kadota M, et al. Pharmacodynamic and pathway analysis of three presumed inhibitors of poly (ADP-ribose) polymerase: ABT888, AZD2281, and BSI201. Cancer Res. 2011;71:4527-4527.

38. Patel AG, De Lorenzo SB, Flatten KS, Poirier GG, Kaufmann SH. Failure of iniparib to inhibit poly(ADP-Ribose) polymerase in vitro. Clin Cancer Res. 2012;18(6):1655-1662.

39. Liu X, Shi Y, Maag DX, et al. Iniparib nonselectively modifies cysteinecontaining proteins in tumor cells and is not a bona fide PARP inhibitor. Clin Cancer Res. 2012;18(2):510-523.

40. Pierce A, McGowan PM, Cotter M, et al. Comparative antiproliferative effects of iniparib and olaparib on a panel of triple-negative and non-triple-negative breast cancer cell lines. Cancer Biol Ther. 2013; 14(6):537-545.

41. Rugo H, Olopade O, DeMichele A, et al. Abstract s5-02: Veliparib/carboplatin plus standard neoadjuvant therapy for high-risk breast cancer: first efficacy results from the I-SPY 2 trial. Cancer Res. 2013;73:S5-S2.

42. Bouwman P, Jonkers J. Molecular pathways: how can BRCA-mutated tumors become resistant to PARP inhibitors? Clin Cancer Res. 2014; 20(3):540-547.

43. Fox E, Bates SE. Tariquidar (XR9576): a P-glycoprotein drug efflux pump inhibitor. Expert Rev Anticancer Ther. 2007;7(4):447-459.

44. US National Library of Medicine. Clinical Trials. gov [webpage on the Internet]. Trial of Olaparib in Combination with AZD5363 (ComPAKT) (ComPAKT). Available from: https:/www.clinicaltrials.gov/ct2/show/ NCT02338622?term=TNBC+PARP\&rank=2. Accessed July 6, 2016.

45. Dent RA, Lindeman GJ, Clemons M, et al. Phase I trial of the oral PARP inhibitor olaparib in combination with paclitaxel for first-or second-line treatment of patients with metastatic triple-negative breast cancer. Breast Cancer Res. 2013;15(5):R88.

46. US National Library of Medicine. Clinical Trials. gov [webpage on the Internet]. PARP Inhibition for Triple Negative Breast Cancer (ER-/PR-/HER2-) With BRCA1/2 Mutations. Available from: https:// clinicaltrials.gov/ct2/show/NCT01074970?term=NCT01074970\&ran $\mathrm{k}=1$. Accessed July 6, 2016.

47. US National Library of Medicine. Clinical Trials. gov [webpage on the Internet]. A Study Evaluating Safety and Efficacy of the Addition of ABT-888 Plus Carboplatin Versus the Addition of Carboplatin to Standard Chemotherapy Versus Standard Chemotherapy in Subjects With Early Stage Triple Negative Breast Cancer. Available from: https:/www. clinicaltrials.gov/ct2/show/NCT02032277?term=NCT02032277\& rank=1. Accessed July 6, 2016. 
48. US National Library of Medicine. Clinical Trials. gov [webpage on the Internet]. Two Regimens of SAR240550/Weekly Paclitaxel and Paclitaxel Alone as Neoadjuvant Therapy in Triple Negative Breast Cancer Patients (SOLTI NEOPARP). Available from: https://www. clinicaltrials.gov/ct2/show/NCT01204125?term=TNBC+PARP\& rank=1. Accessed July 6, 2016.

49. O'Shaughnessy J, Schwartzberg LS, Danso MA, et al. A randomized phase III study of iniparib (BSI-201) in combination with gemcitabine/ carboplatin (G/C) in metastatic triple-negative breast cancer (TNBC). J Clin Oncol. 2011;29:1007.

50. Byrski T, Huzarski T, Dent R, et al. Pathologic complete response to neoadjuvant cisplatin in BRCA1-positive breast cancer patients. Breast Cancer Res Treat. 2014;147(2):401-405.

51. Perou CM. Molecular stratification of triple-negative breast cancer. Oncologist. 2010;15:39-48.

52. Petrelli F, Coinu A, Borgonovo K, et al. The value of platinum agents as neoadjuvant chemotherapy in triple-negative breast cancers: a systematic review and meta-analysis. Breast Cancer Res Treat. 2014;144(2): 223-232.

53. O'shaughnessy J, Osborne C, Pippen J, et al. Efficacy of BSI-201, a poly (ADP-ribose) polymerase-1 (PARP1) inhibitor, in combination with gemcitabine/carboplatin $(\mathrm{G} / \mathrm{C})$ in patients with metastatic triplenegative breast cancer (TNBC): results of a randomized phase II trial. J Clin Oncol. 2009;27:3.

54. O'Shaughnessy J, Osborne C, Pippen JE, et al. Iniparib plus chemotherapy in metastatic triple-negative breast cancer. $N$ Engl $J$ Med. 2011;364(3):205-214.

55. Telli ML, Jensen KC, Vinayak S, et al. Phase II study of gemcitabine, carboplatin, and iniparib as neoadjuvant therapy for triple-negative and BRCA1/2 mutation-associated breast cancer with assessment of a tumor-based measure of genomic instability: PrECOG 0105. J Clin Oncol. 2015;33:1895-1901.

56. von Minckwitz G, Schneeweiss A, Loibl S, et al. Neoadjuvant carboplatin in patients with triple-negative and HER2-positive early breast cancer (GeparSixto; GBG 66): a randomised phase 2 trial. Lancet Oncol. 2014;15(7):747-756.

57. US National Library of Medicine. Clinical Trials. gov [webpage on the Internet]. Debio 1143 in Combination With Carboplatin and Paclitaxel in Patient With Advanced Solid Malignancies. Available from: https:// www.clinicaltrials.gov/ct2/show/NCT01930292?term=platinum+ TNBC\&rank=2. Accessed July 6, 2016.

58. US National Library of Medicine. Clinical Trials. gov [webpage on the Internet]. Cisplatin vs Paclitaxel for Triple Neg. Available from: https:// www.clinicaltrials.gov/ct2/show/NCT01982448?term=platinum+ TNBC\&rank=3. Accessed July 6, 2016.

59. US National Library of Medicine. Clinical Trials. gov [webpage on the Internet]. Predictors of Response to Neoadjuvant Docetaxel-Carboplatin Chemotherapy for Patients With Stage II and III Triple Negative Breast Cancer. Available from: https://www.clinicaltrials.gov/ct2/show/NCT 01560663?term=platinum+TNBC\&rank=4. Accessed July 6, 2016.

60. US National Library of Medicine. Clinical Trials. gov [webpage on the Internet]. Romidepsin Plus Cisplatin in Locally Recurrent or Metastatic Triple Negative Breast Cancer (TNBC). Available from: https://www. clinicaltrials.gov/ct2/show/NCT02393794?term=platinum+TNBC\& rank=5. Accessed July 6, 2016

61. US National Library of Medicine. Clinical Trials. gov [webpage on the Internet]. Efficacy and Safety Study of Neoadjuvant Chemotherapy for Local Advanced Triple Negative Breast Cancer Patients. Available from: https://www.clinicaltrials.gov/ct2/show/NCT01216124?term= platinum+TNBC\&rank=6. Accessed July 6, 2016.

62. US National Library of Medicine. Clinical Trials. gov [webpage on the Internet]. Comparison Study of Neoadjuvant Paclitaxel Plus Carboplatin/ Epirubicin Treatment in Triple-negative Breast Cancer. Available from: https://www.clinicaltrials.gov/ct2/show/NCT01276769? term=platinum+TNBC\&rank=7. Accessed July 6, 2016.
63. US National Library of Medicine. Clinical Trials. gov [webpage on the Internet]. Comparison Study of Adjuvant Chemotherapy for Chinese Triple Negative Breast Cancer. Available from: https://www. clinicaltrials.gov/ct2/show/NCT01216111?term=platinum+TNBC\& rank $=8$. Accessed July 6, 2016.

64. US National Library of Medicine. Clinical Trials. gov [webpage on the Internet]. Available from: https://www.clinicaltrials.gov/ct2/results? term=platinum+TNBC\&Search=Search. Accessed July 6, 2016.

65. Sikov WM, Berry DA, Perou CM, et al. Impact of the addition of carboplatin and/or bevacizumab to neoadjuvant once-per-week paclitaxel followed by dose-dense doxorubicin and cyclophosphamide on pathologic complete response rates in stage II to III triple-negative breast cancer: CALGB 40603 (Alliance). J Clin Oncol. 2015;33:13-21.

66. Rakha EA, El-Sayed ME, Green AR, Lee AH, Robertson JF, Ellis IO. Prognostic markers in triple-negative breast cancer. Cancer. 2007; 109(1):25-32.

67. Carey LA, Rugo HS, Marcom PK, et al. TBCRC 001: randomized phase II study of cetuximab in combination with carboplatin in stage IV triple-negative breast cancer. J Clin Oncol. 2012;30(21): $2615-2623$.

68. Corkery B, Crown J, Clynes M, O’Donovan N. Epidermal growth factor receptor as a potential therapeutic target in triple-negative breast cancer. Ann Oncol. 2009;20(5):862-867.

69. Elbaz M, Nasser MW, Ravi J, et al. Modulation of the tumor microenvironment and inhibition of EGF/EGFR pathway: novel antitumor mechanisms of cannabidiol in breast cancer. Mol Oncol. 2015; 9(4):906-919.

70. US National Library of Medicine. Clinical Trials. gov [webpage on the Internet]. Cetuximab and Cisplatin in the Treatment of "Triple Negative" (Estrogen Receptor [ER] Negative, Progesterone Receptor [PgR] Negative, and Human Epidermal Growth Factor Receptor 2 [HER2] Negative) Metastatic Breast Cancer (BALI-1). Available from: https://www.clinicaltrials.gov/ct2/show/NCT00463788?term=NCT00 463788.\&rank=1. Accessed July 6, 2016.

71. US National Library of Medicine. Clinical Trials. gov [webpage on the Internet]. Cetuximab With or Without Carboplatin in Treating Women With Estrogen Receptor-Negative, Progesterone Receptor-Negative Metastatic Breast Cancer. Available from: https://www.clinicaltrials. gov/ct2/show/NCT00232505?term=NCT00232505.\&rank=1. Accessed July 6, 2016.

72. US National Library of Medicine. Clinical Trials. gov [webpage on the Internet]. Neo-Adjuvant Study in Triple Negative Breast Cancer Patients (ICE). Available from: https://www.clinicaltrials.gov/ct2/show/ NCT01097642?term=cetuximab+TNBC\&rank=1. Accessed July 6, 2016.

73. US National Library of Medicine. Clinical Trials. gov [webpage on the Internet]. Pilot Study of Veliparib (ABT-888) and Lapatinib (Tykerb) in Patients with Metastatic, Triple Negative Breast Cancer. Available from: https://www.clinicaltrials.gov/ct2/show/NCT02158507?term=1 apatinib+TNBC\&rank=1. Accessed July 6, 2016.

74. US National Library of Medicine. Clinical Trials. gov [webpage on the Internet]. The Safety and Effects of Gefitinib in Triple-negative, EGFR Positive Metastatic Breast Cancer. Available from: https://www. clinicaltrials.gov/ct2/show/NCT01732276?term=gefitinib+TNBC\& rank=1. Accessed July 6, 2016.

75. US National Library of Medicine. Clinical Trials. gov [webpage on the Internet]. Panitumumab, Gemcitabine and Carboplatin in TripleNegative Metastatic Breast Cancer. Available from: https://www. clinicaltrials.gov/ct2/show/NCT00894504?term $=$ NCT00894504\& rank=1. Accessed July 6, 2016.

76. US National Library of Medicine. Clinical Trials. gov [webpage on the Internet]. Addition of Carboplatin to Neoadjuvant Therapy for Triplenegative and HER2-positive Early Breast Cancer (GeparSixto). Available from: https://www.clinicaltrials.gov/ct2/show/NCT01426880? term=lapatinib+TNBC\&rank=2. Accessed July 6, 2016. 
77. US National Library of Medicine. Clinical Trials. gov [webpage on the Internet]. A Phase 2 Trial of Standard Chemotherapy, With or Without BSI-201, in Patients With Triple Negative Metastatic Breast Cancer. Available from: https:/www.clinicaltrials.gov/ct2/show/NCT00540 358 ?term $=$ Epidermal+growth + factor + receptor + as $+\mathrm{a}+$ potential + ther apeutic + target + in + triple-negative + breast + cancer $\&$ rank $=5$. Accessed July 6, 2016.

78. Andre F, Job B, Dessen P, et al. Molecular characterization of breast cancer with high-resolution oligonucleotide comparative genomic hybridization array. Clin Cancer Res. 2009;15(2):441-451.

79. Linderholm BK, Hellborg H, Johansson U, et al. Significantly higher levels of vascular endothelial growth factor (VEGF) and shorter survival times for patients with primary operable triple-negative breast cancer. Ann Oncol. 2009;20(10):1639-1646.

80. Miles DW, Chan A, Dirix LY, et al. Phase III study of bevacizumab plus docetaxel compared with placebo plus docetaxel for the first-line treatment of human epidermal growth factor receptor 2-negative metastatic breast cancer. J Clin Oncol. 2010;28:3239-3247.

81. Robert NJ, Diéras V, Glaspy J, et al. RIBBON-1: randomized, doubleblind, placebo-controlled, phase III trial of chemotherapy with or without bevacizumab for first-line treatment of human epidermal growth factor receptor 2-negative, locally recurrent or metastatic breast cancer. J Clin Oncol. 2011;29:1252-1260.

82. Makhoul I, Klimberg VS, Korourian S, et al. Combined neoadjuvant chemotherapy with bevacizumab improves pathologic complete response in patients with hormone receptor negative operable or locally advanced breast cancer. Am J Clin Oncol. 2015;38(1):74-79.

83. Gerber B, Loibl S, Eidtmann H, et al; German Breast Group Investigators. Neoadjuvant bevacizumab and anthracycline-taxane-based chemotherapy in 678 triple-negative primary breast cancers; results from the geparquinto study (GBG 44). Ann Oncol. 2013;24(12):2978-2984.

84. Brufsky A, Valero V, Tiangco B, et al. Impact of bevacizumab (BEV) on efficacy of second-line chemotherapy (CT) for triple-negative breast cancer (TNBC): analysis of RIBBON-2. J Clin Oncol. 2011;29:1010.

85. Stanton SE. Triple-negative breast cancer: immune modulation as the new treatment paradigm. Am Soc Clin Oncol. 2015:e25-e30.

86. Demaria S, Volm MD, Shapiro RL, et al. Development of tumorinfiltrating lymphocytes in breast cancer after neoadjuvant paclitaxel chemotherapy. Clin Cancer Res. 2001;7(10):3025-3030.

87. Wimberly H, Brown JR, Schalper KA, et al. PD-L1 expression correlates with tumor-infiltrating lymphocytes and response to neoadjuvant chemotherapy in breast cancer. Cancer Immunol Res. 2015;3(4):326-332.

88. Pardoll DM. The blockade of immune checkpoints in cancer immunotherapy. Nat Rev Cancer. 2012;12(4):252-264.

89. Emens LA, Braiteh FS, Cassier P, et al. Inhibition of PD-L1 by MPDL3280A leads to clinical activity in patients with metastatic triplenegative breast cancer (TNBC). Cancer Res. 2015;75:2859-2859.

90. Barrett MT, Anderson KS, Lenkiewicz E, et al. Genomic amplification of 9p24.1 targeting JAK2, PD-L1, and PD-L2 is enriched in high-risk triple negative breast cancer. Oncotarget. 2015;6(28): 26483-26493.

91. Adams S, Diamond J, Hamilton E, et al. Safety and clinical activity of atezolizumab (anti-PDL1) in combination with nab-paclitaxel in patients with metastatic triple-negative breast cancer. Proceedings of the Thirty-Eighth Annual CTRC-AACR San Antonio Breast Cancer Symposium. 2015; 6-10.

92. Cimino-Mathews A, Foote JB, Emens LA. Immune targeting in breast cancer. Oncology. 2015;29:375-375.

93. Denkert C, Von Minckwitz G, Brase JC, et al. Tumor-infiltrating lymphocytes and response to neoadjuvant chemotherapy with or without carboplatin in human epidermal growth factor receptor 2-positive and triple-negative primary breast cancers. J Clin Oncol. 2015;33:983-991.

94. Tung NM, Winer EP. Tumor-infiltrating lymphocytes and response to platinum in triple-negative breast cancer. J Clin Oncol. 2015; 33:969-971.
95. Issa-Nummer Y, Darb-Esfahani S, Loibl S, et al. Prospective validation of immunological infiltrate for prediction of response to neoadjuvant chemotherapy in HER2-negative breast cancer-a substudy of the neoadjuvant GeparQuinto trial. PLoS One. 2013;8:e79775.

96. Yamaguchi R, Tanaka M, Yano A, et al. Tumor-infiltrating lymphocytes are important pathologic predictors for neoadjuvant chemotherapy in patients with breast cancer. Hum Pathol. 2012;43(10):1688-1694.

97. Craig DW, O'Shaughnessy JA, Kiefer JA, et al. Genome and transcriptome sequencing in prospective metastatic triple-negative breast cancer uncovers therapeutic vulnerabilities. Mol Cancer Ther. 2013; 12(1):104-116

98. Shrivastava S, Jeengar MK, Reddy VS, Reddy GB, Naidu VG. Anticancer effect of celastrol on human triple negative breast cancer: possible involvement of oxidative stress, mitochondrial dysfunction, apoptosis and PI3K/Akt pathways. Exp Mol Pathol. 2015;98(3):313-327.

99. Choi JE, Kang SH, Lee SJ, Bae YK. Androgen receptor expression predicts decreased survival in early stage triple-negative breast cancer. Ann Surg Oncol. 2015;22(1):82-89.

100. US National Library of Medicine. Clinical Trials. gov [webpage on the Internet]. Bicalutamide in Treating Patients With Metastatic Breast Cancer. Available from: https:/clinicaltrials.gov/ct2/show/NCT0046 8715?term=NCT00468715\&rank=1. Accessed July 6, 2016.

101. Liu X, Gomez-Pinillos A, Ferrari AC. Simultaneous targeting of the androgen receptor and $\mathrm{PI} 3 \mathrm{~K} / \mathrm{mTOR}$ pathway in androgen-dependent and androgen-independent prostate cancer cells. J Clin Oncol. 2010;28:e15049.

102. Gu G, Gelsomino L, Covington KR, et al. Targeting thyroid hormone receptor beta in triple-negative breast cancer. Breast Cancer Res Treat. 2015;150(3):535-545.

103. Taliaferro-Smith L, Oberlick E, Liu T, et al. FAK activation is required for IGF1R-mediated regulation of EMT, migration, and invasion in mesenchymal triple negative breast cancer cells. Oncotarget. 2015;6(7):4757-4772.

104. Cerqueira OL, Truesdell P, Baldassarre T, et al. CIP4 promotes metastasis in triple-negative breast cancer and is associated with poor patient prognosis. Oncotarget. 2015;6(11):9397-9408.

105. Tate CR, Rhodes LV, Segar HC, et al. Targeting triple-negative breast cancer cells with the histone deacetylase inhibitor panobinostat. Breast Cancer Res. 2012;14(3):R79.

106. Nanda R, Chow LQ, Dees EC, et al. Abstract S1-09: a phase Ib study of pembrolizumab (MK-3475) in patients with advanced triple-negative breast cancer. Cancer Res. 2015;75:S1-S09.

107. Blanchard Z, Paul BT, Craft B, ElShamy WM. BRCA1-IRIS inactivation overcomes paclitaxel resistance in triple negative breast cancers. Breast Cancer Res. 2015;17:5.

108. Tomao F, Papa A, Zaccarelli E, et al. Triple-negative breast cancer: new perspectives for targeted therapies. Onco Targets Ther. 2015;8:177-193.

109. Rogers LQ, Courneya KS, Anton PM, et al. Effects of the BEAT Cancer physical activity behavior change intervention on physical activity, aerobic fitness, and quality of life in breast cancer survivors: a multicenter randomized controlled trial. Breast Cancer Res Treat. 2015;149(1):109-119.

110. Swisher AK, Abraham J, Bonner D, et al. Exercise and dietary advice intervention for survivors of triple-negative breast cancer: effects on body fat, physical function, quality of life, and adipokine profile. Support Care Cancer. 2015;23(10):2995-3003.

111. Abdulkarim BS, Cuartero J, Hanson J, Deschênes J, Lesniak D, Sabri S. Increased risk of locoregional recurrence for women with T1-2N0 triple-negative breast cancer treated with modified radical mastectomy without adjuvant radiation therapy compared with breast-conserving therapy. J Clin Oncol. 2011;29(21):2852-2858.

112. Untch M, Konecny GE, Paepke S, von Minckwitz G. Current and future role of neoadjuvant therapy for breast cancer. Breast. 2014; 23(5):526-537. 


\section{Publish your work in this journal}

OncoTargets and Therapy is an international, peer-reviewed, open access journal focusing on the pathological basis of all cancers, potential targets for therapy and treatment protocols employed to improve the management of cancer patients. The journal also focuses on the impact of management programs and new therapeutic agents and protocols on

patient perspectives such as quality of life, adherence and satisfaction. The manuscript management system is completely online and includes a very quick and fair peer-review system, which is all easy to use. Visit http://www.dovepress.com/testimonials.php to read real quotes from published authors.

Submit your manuscript here: http://www.dovepress.com/oncotargets-and-therapy-journal 